\title{
Staphylococcus Epidermidis Producteur D'entérotoxine C Impliqué Dans Une Septicémie À Foyer Urinaire
}

\section{Chimène Nadège Mahoussi Nanoukon, Msc.}

Université d'Abomey-Calavi,

Laboratoire de Biologie et de Typage Moléculaire en Microbiologie, Benin.

Université de Strasbourg,

EA7290 Virulence Bactérienne Précoce, Institut de Bactériologie, 3 rue

Koeberlé,

F-67000 Strasbourg, Fédération de Médecine Translationnelle de Strasbourg,

CHRU Strasbourg, France

Euloge Gbètondji Dassou, Chirurgien Urologue-Andrologue

Marcos Dousso, Pédiatre

Frédéric Sogbo, Interne des hôpitaux

Centre National Hospitalier et Universitaire Hubert Koutoukou Maga

(CNHU-HKM) de Cotonou, Bénin

Haziz Sina, PhD

Université d'Abomey-Calavi,

Laboratoire de Biologie et de typage Moléculaire en Microbiologie, Benin

Gilles Prevost, PhD

Université de Strasbourg,

EA7290 Virulence Bactérienne Précoce, Institut de Bactériologie, 3 rue Koeberlé, F-67000 Strasbourg, Fédération de Médecine Translationnelle de Strasbourg, CHRU Strasbourg, France

\section{Lamine Baba-Moussa, PhD}

Université d'Abomey-Calavi,

Laboratoire de Biologie et de Typage Moléculaire en Microbiologie, Benin doi: 10.19044/esj.2017.v13n24p278 URL:http://dx.doi.org/10.19044/esj.2017.v13n24p278

\begin{abstract}
In developing countries, coagulase-negative staphylococcal (SCN) infections are often neglected. Here, we describe the identification of a coagulase-negative staphylococcal strain secreting type C staphylococcal enterotoxin (SEC) in a field weakened by severe malaria in a 12-year-old child and summarize the clinical characteristics of the patient. The identification of the strain is carried out by MALDI-TOF mass spectrometry
\end{abstract}


after protein extraction. The toxigenic capacity of the species in question is determined by the polymerase chain reaction (PCR) and the radial immunoprecipitation method. The patient's medical record was used for the study of the correlation between the clinical phenotype of the patient and the production of SEC by the strain. S. epidermidis was identified as the pathogen responsible of bacteremia which induces clinical sepsis, hematemesis and thrombocytopenia. The PCR test revealed that the isolated strain was positive for sec gene and its expression was confirmed by the radial immunoprecipitation assay. The study of the patient's medical record shows that the enterotoxin produced by the strain may have contributed to the severity of the clinical picture with the presence of disseminated intravascular coagulation stigmata (DIC) in a context of sepsis with a urinary starting point. These data suggest that CNS infections may be underestimated due to difficulties in species identification and the toxigenic capacity of some of these isolates.

Keywords: Staphylococcus epidermidis, septicemia, enterotoxins; mass spectrometry

\section{Résumé}

Dans les pays en voie de développement les infections à staphylocoque à coagulase négative $(\mathrm{SCN})$ sont souvent négligées. Ici, nous décrivons l'identification d'une souche de $\mathrm{SCN}$ sécrétrice d'entérotoxine staphylococcique de type C (SEC) sur un terrain fragilisé par un paludisme grave chez un enfant de 12 ans et résumons les caractéristiques clinique du patient. L'identification de la souche est réalisée par la spectrométrie de masse MALDI-TOF après une extration protéique. La capacité toxigénique de l'espèce en cause est déterminée par la réaction de polymérisation en chaine de (PCR) et par le test d'immunoprécipitation radiale. Le dossier médical du patient est exploité pour l'étude de la corrélation entre le phénotype clinique du patient et la production de SEC par la souche. $S$. epidermidis a été identifié comme l'agent pathogène responsable de la bactériémie ayant induit un sepsis clinique, une hématémèse et une thrombopénie. Le test de PCR a révélé que la souche était positive pour le gène sec dont l'expression a été confirmée par le test d'immunoprécipitation radiale d'Ouctchterlony. L'étude du dossier médical du patient montre que l'entérotoxine produite par la souche à bien pu contribuer à la gravité du tableau clinique avec la présence de stigmates de coagulation intravasculaire disséminée (CIVD) dans un contexte de sepsis à point de départ urinaire. Ces données suggèrent que les infections à SCN peuvent être sous estimées à cause des difficultés d'identification au niveau espèce et la capacité toxigène de certains de ces isolats. 
Mots clés: Staphylococcus epidermidis, septicémie, entérotoxine, spectrométrie de masse

\section{Introduction}

Les staphylocoques à coagulase négative $(\mathrm{SCN})$ sont des bactéries commensales de la peau à l'origine d'infections opportunistes le plus souvent nosocomiales. Une relation directe a récemment été rapportée entre les accès palustres chez les enfants vivant en zone d'endémie et de telles infections opportunistes (Scott et al., 2011). Cette situation participe probablement au fort taux de mortalité observé chez les enfants présentant un paludisme grave en Afrique sub-saharienne (Were et al., 2011). Les pathogènes les plus fréquemment isolés des hémocultures chez de tels patients sont les Salmonella non-typhi, les entérobactéries, et, plus rarement, Staphylococcus aureus (Bronzan et al., 2007). Bien que les staphylocoques à coagulase négative soient habituellement considérés comme des pathogènes faiblement virulents, plusieurs études ont rapporté l'identification de facteurs de virulence chez certaines espèces. En effet, sachant la proximité génétique des espèces de staphylocoques et la cohabitation des SCN avec $S$. aureus sur la peau et les muqueuses de l'homme, les facteurs de virulence habituellement produits par S. aureus pourraient être retrouvés chez les autres staphylocoques moins pathogènes par des mécanismes de transfert horizontal de gène de virulence. Par exemple, les entérotoxines staphylococciques ayant des propriétés pyrogénique, endotoxique et superantigènique originairement isolées chez $S$. aureus, ont été récemment identifiées chez des souches de SCN d'origine humaine (Madhusoodanan et al., 2007; Nanoukon et al., 2016). Chez $S$. aureus, ces toxines sont associées à plusieurs pathologies dont la plus fréquente est l'intoxication alimentaires qui est due à l'ingestion d'une ou plusieurs entérotoxines staphylococciques préalablement développées dans l'aliment (Spaulding, 2013). Le syndrome de choc toxique staphylococcique est provoqué par la diffusion dans l'organisme de certains superantigènes dont les entérotoxines staphylococciques (B, C, etc.) (McCormick et al., 2001). La production d'entérotoxines staphylococciques à la phase aiguë a été aussi incriminée dans la genèse de la maladie de Kawasaki (Prévost, 2004). En ce qui concerne les SCN, il n'y presque pas d'étude ayant permis d'établir une corrélation clinico-biologique entre l'expression d'entérotoxine stapylococcique et les manisfestations cliniques pouvant expliquer la pathogénicité des souches.

La présente étude décris l'identification d'une souche de $S$. epidermidis productrice d'entérotoxine staphylococcique $\mathrm{C}$, impliquée dans une bactériémie associée à un paludisme grave et rapporte les caractéristiques cliniques du patient. 


\section{Matériels et méthodes}

\section{Collecte des échantillons}

L'étude a enrôlé un enfant de sexe masculin de l'âge de 12 ans qui a été admis en urgence au Centre National Hospitalier et Universitaire Hubert Koutoukou Maga (CNHU-HKM) de Cotonou avec des signes de septicémie à foyer urinaire. Les échantillons de sang et d'urine de ce patient ont été collectés pour analyses. Les analyses microbiologiques ont été réalisées au Laboratoire de Microbiologie dudit hôpital.

\section{Isolement et identification des SCN}

Le sang ( $3 \mathrm{ml})$ a été prélevé à l'admission du patient, aux services des urgences dans des flacons d'hémoculture (BD BACTEC-plus ${ }^{\mathrm{TM}}$ ) contenant du bouillon de sodja enrichi et du $\mathrm{CO}_{2}$, avant toute antibiothérapie et au moment de l'élévation thermique. Les cultures positives après 24-48 h d'incubation sont soumises à la coloration de Gram et à la culture sur des milieux usuels. L'identification phénotypique de la souche isolée de l'hémoculture du patient a été réalisée par des méthodes standards incluant les tests biochimiques. Brièvement, la souche a été d'abord testée pour la recherche de la production de la staphylocoagulase libre en utilisant le plasma lyophilisé de lapin (Bio-Rad Laboratories, Marne-La-Coquette, France) (Riegel et al., 2006) et la recherche de la coagulase liée avec le test d'agglutination au latex Pastorex Staph-Plus (Bio-Rad Laboratories) (Riegel et al., 2006).

L'examen cytobactériologique des urines (ECBU) a été réalisé à la suite d'un prélèvement aseptique des urines. Elles sont ensuite ensemencées sur les géloses Chapman et la gélose au sang (GS). Enfin, la coloration Gram de la souche soupçonnée a été réalisée suivi d'un test de recherche de la staphylocoagulase libre.

Après la négativité de ces tests, l'isolat a été considéré comme un staphylocoque à coagulase négative et a été ensuite identifiée au niveau espèce à la spectrométrie de masse MALDI-TOF avec un MALDI-TOF Microflex LT/LRF couplé à l'algorithme de biotypage MALDI à l'Institut de Bactériologie de l'université de Strasbourg selon les recommandations du fabriquant. Un score d'identification $\geq 2$ est considéré comme une forte confiance d'identification au niveau espèce de la souche.

\section{Susceptibilité aux antibiotiques}

La suceptibilité de la souche aux antibiotiques a été recherchée avec Vitek 2 selon les recommandations du comité Européen sur le test de suceptibilité antimicrobien au cours de la période d'étude (http://www.eucast.org/). Les antibiotoques testés sont les suivants : Céfoxitine $(10 \mu \mathrm{g})$, Benzylpénicilline $(6 \mu \mathrm{g})$, Oxacilline $(1 \mu \mathrm{g})$, Gentamicine 
$(10 \mu \mathrm{g})$, Kanamicine $(30 \mu \mathrm{g})$, Tobramicine $(10 \mu \mathrm{g})$, Ofloxacine $(5 \mu \mathrm{g})$, Clindamycine $(2 \mu \mathrm{g})$, Erythromycine $(15 \mu \mathrm{g})$, Lincomycine $(15 \mu \mathrm{g})$, Pristinamycine $(15 \mu \mathrm{g})$, Linézoide $(30 \mu \mathrm{g})$, Téicoplanine $(30 \mu \mathrm{g})$, Vancomycine $(30 \mu \mathrm{g})$, Tétracycline $(30 \mu \mathrm{g})$, Fosfomycine $(200 \mu \mathrm{g})$, Nitrofurantoine $(300 \mu \mathrm{g})$, Acide fusidique $(10 \mu \mathrm{g})$, Rifampicine $(5 \mu \mathrm{g})$, Triméthoprime-sulfamides $(1.25 / 23.75 \mu \mathrm{g})$.

\section{Production de toxines}

La recherche de gène codant les entérotoxines staphylococciques (SEA, SEB, SEC, SEG, SEH), la Leucocidine de Panton et Valentine (LPV) (LukF-PV, LukS-PV) et la gamma-hémolysine de S. aureus a été réalisée par la réaction de polymérisation en chaine avec les oligonucléotides spécifiques de $S$. aureus.

Enfin l'expression du gène présent est recherchée par la technique d'immunoprécipitation radiale d'Ouctchterlony en utilisant les anticorps purifiés de lapin spécifique aux toxines de S. aureus (Gravet et al., 2011). Pour cette detection, $40 \mu \mathrm{l}$ de surnageant de culture $(113 \mathrm{ng} / \mu \mathrm{l})$, de toxine $(100 \mu \mathrm{g} / \mathrm{mL})$ et d'anticorps $(2,25 \mu \mathrm{g} / \mathrm{mL})$ sont déposés dans les puits correspondants, sur un gel d'agarose de $0,6 \%$. Après $24 \mathrm{~h}$ incubation à $4^{\circ} \mathrm{C}$, la présence de SEC dans le surnageant est mise en évidence par l'apparition d'un arc de précipitation entre le puits contenant l'anticorps anti-SEC et le puits contenant le surnageant de culture de $S$. epidermidis.

\section{Importance microbiologique}

L'importance microbiologique de la souche isolée de l'hémoculture et de l'ECBU a été évaluée selon les recommandations du manuel Européen des Critères de Microbiologie Clinique pour l'Interprétation (EMCM, 2016). Ainsi, pour l'hémoculture, la souche de SCN est considérée comme responsable de l'infection s'il y a au moins deux échantillons positifs pour la même espèce à des moments de cultures différentes. Pour les échantillons d'urines, la bactériurie est significative à une valeur $\geq 10^{5} \mathrm{CFU} / \mathrm{ml}$, lorsqu'elle est associée à une leucocyurie $\geq 10^{4} \mathrm{CFU} / \mathrm{ml}$.

\section{Données cliniques}

Le dossier médical du patient obtenu au service pédiatrique du CNHU-HKM a été consulté avec un regard sur les données cliniques incluant les conditions l'admission aux Urgences, les résultats d'hémogramme, de la C-protéine réactive (CRP) et de la densité parasitaire déterminés au Laboratoire d'Hématologie, de Biochimie et de Parasitologie selon les procédures standards. 


\section{Résultats}

\section{Données cliniques}

Cette étude porte sur un patient de 12 ans qui est admis le 12 novembre 2015 au service d'accueil des urgences du CNHU-HKM pour une fièvre évoluant depuis 10 jours et associée à une hémorragie digestive haute et basse. L'état septique est d'emblée inquiétant avec une fréquence cardiaque à $120 \mathrm{bpm}$, une polypnée à 35 cycles par minute pour une fièvre à $40^{\circ} \mathrm{C}$. Biologiquement le patient présente une anémie à $11,7 \mathrm{~g} / \mathrm{dl}$, une hyperleucocytose à $12 \times 10^{9} / 1$ dont $10,2 \times 10^{9} / 1$ de polynucléaires neutrophiles. Les plaquettes sont basses à $21 \times 10^{9} / 1$ et le taux de prothrombine (TP) spontanément à $64 \%$ avec un temps de Céphaline Kaolin (TCK) normal. Le frottis et la goutte épaisse contiennent Plasmodium falciparum avec une densité parasitaire de 312941 trophozoites/ $\mu 1$ de sang. Les hémocultures réalisées à l'admission objectivent la présence de cocci à Gram $(+)$ et l'examen cytobactériologique des urines montre une leucocyturie pathologique. Le patient est traité en urgence par artésunate associé à une antibiothérapie par ceftriaxone. L'identification bactérienne réalisée au deuxième jour retrouve un Staphylococcus epidermidis dans le sang et dans les urines avec un antibiogramme identique: sensibilité à Méticilline et donc à l'ensemble des $\beta$-lactamines, aux aminosides, au linézolide, aux sulfaméthoxazole/triméthroprime et une résistance à la fosfomycine. L'identification de ce staphylocoque est confirmée avec un score d'identification $\geq 2$ à la spectrométrie de masse et par séquençage du gène codant l'ARN 16S. La recherche de coagluase libre ou liée est négative.

\section{Caracterisation de la toxigenicite de la souche}

Le test de la PCR a été positif pour la recherche du gène codant l'entérotoxine staphylococcique C ( $\mathrm{sec}$ ) de $S$. epidermidis (Figure 1) dont l'expression a été confirmée en immunologie par la méthode d'agglutination au latex en utilisant le kit de détection des entérotoxines staphylococcique SEA, SEB, SEC, SED et SEE SET-RPLA-T900, et négatif les gènes sea, $s e b$, seg et seh ainsi que pour le gène codant le clumping factor $\mathrm{b}(\mathrm{clfb})$. 


\section{$\begin{array}{lllllllllll}1 & 2 & 3 & 4 & 5 & 6 & 7 & 8 & 9 & 10 & 11\end{array}$}

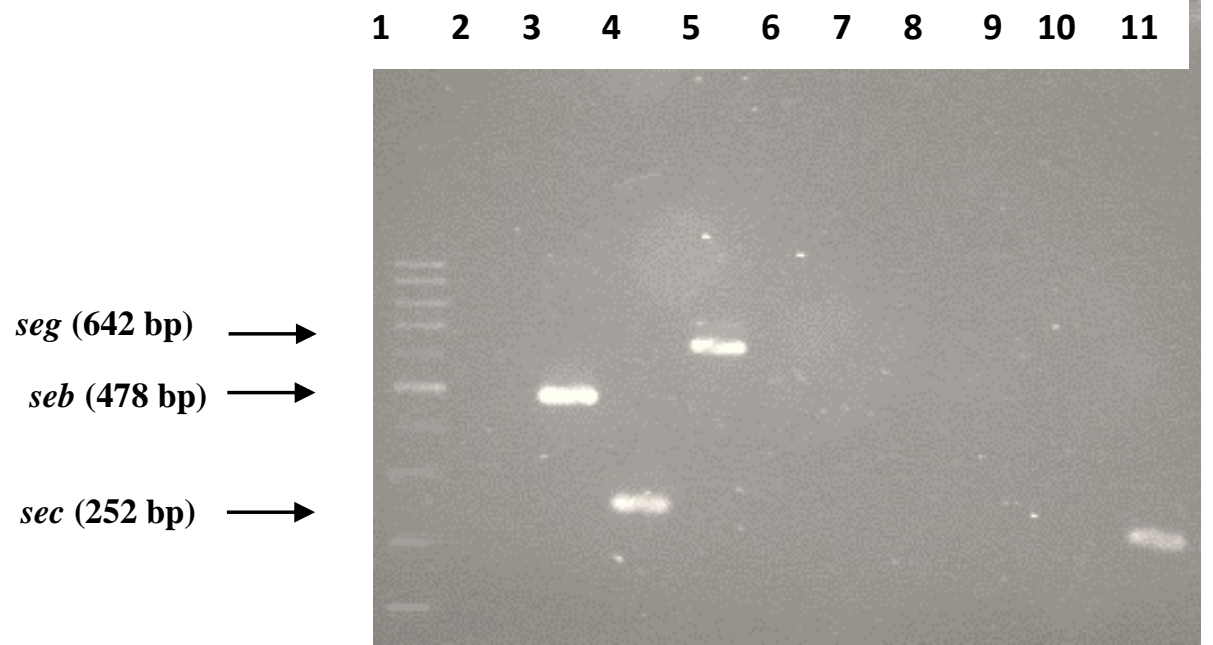

Figure 1: Élèctrophorèse sur gel d'agarose pour la détection du gène sec (252 bp), seg (642) et $s e b$ par PCR chez $S$. epidermidis. Ligne 1: Marqueur de poids moléculaire (100-bp), ligne 2: contrôl négatif, ligne 3: S. aureus SCP FRI 341 (seb +), ligne 4: S. aureus SCP FRI 361 $(\mathrm{sec}+)$, ligne 5, SCP FRI 137 (seg +); ligne 6, 7, 8, 9, et 10: SCN dépourvus des gènes seb, sec, et seg, ligne 11: souche de S. epidermidis positive pour le gène sec.

Le test d'immunoprécipitation radiale d'Ouctchterlony quant à lui a été négatif pour la production de la Leucocidine de Panton et Valentine (LPV : LukF-PV et LukS-PV), et gamma-hémolysines de S. aureus, mais aussi positif avec un anticorps anti-SEC de $S$. aureus (Figure 2).

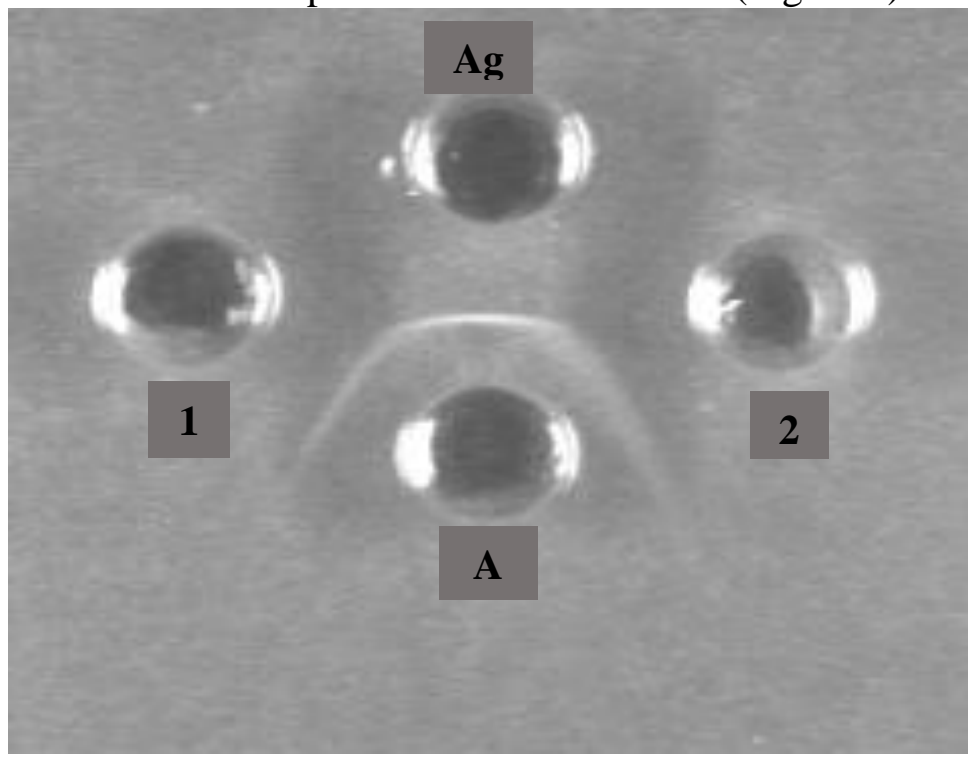

Figure 2: Réaction immunologique du surnageant de culture de $24 \mathrm{~h}$ de $S$. epidermidis (1 et 2) avec l'anticorps de lapin anti-SEC S. aureus SCP FRI 361 (Ac) en présence de la toxine SEC purifiée $(\mathrm{Ag})$, utilisé comme témoin positif 
L'évolution clinique était favorable avec la disparition des signes infectieux et de la CIVD permettant le transfert de ce patient en unité conventionnelle dès le $4^{\text {ème }}$ jour d'hospitalisation. A 3 mois de suivi, le patient ne présentait pas de séquelles ni de récidive infectieuse.

\section{Discussion}

Nous rapportons la production d'entérotoxine staphylococcique C, un des principaux facteurs de virulence habituellement porté par $S$. aureus, par une souche de $S$. epidermidis impliquée dans une bactériémie. La gravité initiale du tableau avec la présence de stigmates de coagulation intravasculaire disséminée (CIVD) ainsi qu'un syndrome hémorragique digestif peuvent être dus tant à l'accès palustre qu'à cette septicémie. L'identification de SCN dans les hémocultures est habituellement considérée comme une simple contamination dans la plupart des études (Were et al., 2011, Sandlund et al., 2013). Mais la survenue dans notre cas d'une dysurie, ainsi que l'identification d'une souche de même espèce et avec le même antibiogramme dans les urines avec production de nitrites nous conduit à confirmer le caractère pathogène de cette souche. Le lien entre accès palustre et septicémie n'est pas clair, mais certains auteurs ont évoqué la possibilité d'un déficit immunitaire transitoire concomitant de ces accès palustres (Nyein et al., 2016). Il faut aussi remarquer que la plupart des infections à $\mathrm{SCN}$ sont nosocomiales, même en cas d'accès palustre alors que dans notre cas cette infection est d'origine communautaire, car tous les prélèvements positifs ont été réalisés à l'admission du patient (Bruneel et al., 2010).

Bien que la capacité des SCN à produire des superantigènes demeure un sujet de débat et que de récentes études ont montré l'absence de production de ces dernières par des souches cliniques de SCN d'origine humaines, la souche isolée dans cette étude est sécrétrice d'entérotoxine et a pu jouer un rôle dans le tableau clinique présenté par ce jeune patient. Cette toxine est à l'origine d'intoxications alimentaires (Balaban et al., 2000) mais récemment, son implication dans des sepsis létaux, des endocardites infectieuses et des lésions rénales aiguës a été démontrée dans un modèle animal (Salgado-Pabón et al., 2013). En effet, l'entérotoxine déclenche directement un effet sur l'épithélium intestinal et sur le nerf vague, provoquant une stimulation du centre émétique et l'augmentation de la motilité intestinale ce qui induit les vomissements observés chez les patients. L'hémorragie digestive présentée par le patient peut être liée au tableau de CIVD présenté dès l'admission et dont la cause peut être ici multiple : accès palustre grave ou bien la septicémie à SCN producteur d'entérotoxine. L'activité de l'entérotoxine peut faciliter la transcytose, permettant à la toxine d'entrer dans la circulation sanguine induisant ainsi l'interaction forcée avec la portion $\mathrm{V} \beta$ des cellules $\mathrm{T}$ et par conséquent, une hyperstimulation des 
lymphocytes T. Cette super stimulation est la cause d'une synthèse et d'une libération excessive de cytokines: Tumor Necrosis Factor- alpha ( $\left.\mathrm{TNF}_{\alpha}\right)$, Interleukin-1 et 2 (IL-1-2) qui agissent comme des vasodilatateurs capillaires, conduisant à une hypotension, un choc, une défaillance multivisécrale et souvent, alors, la mort (McCormick et al., 2001).

\section{Conclusion}

Les infections à $\mathrm{SCN}$ sont souvent négligées, à tort un peu partout dans le monde et particulièrement en Afrique, parce que certaines souches peuvent être virulentes. Notre étude souligne ainsi l'importance de continuer à rechercher la production d'entérotoxines chez $S$. epidermidis qui ne constitue probablement pas un pathogène opportuniste banal.

\section{Soutien financier}

Ce travail a été financé par une bourse de Mérite de la Banque Islamique du Développement et soutenu à la fois par 1'EA7290 Virulence Bactérienne Précoce de la Faculté de Médecine de l'Université de Strasbourg et par le laboratoire de Bactériologie du Centre National Hospitalier et Universitaire Hubert Koutoukou-Maga de Cotonou, Benin.

\section{References:}

1. Bronzan, R. N., Taylor, T. E., Mwenechanya, J., Tembo, M., Kayira, K., Bwanaisa, L.,_Njobvu, A., Kondowe, W., Chalira, C., Walsh, A. L., Phiri, A., Wilson, L. K., Molyneux, M. E. \& Graham, S. M. (2007). Bacteremia in Malawian children with severe malaria: prevalence, etiology, HIV coinfection, and outcome. Journal of Infectious Diseases, 195 (6), 895-904. DOI: 10.1086/511437

2. Balaban, N. \& Rasooly, A. (2000). Staphylococcal enterotoxins. International Journal of Food Microbiology, 61 (1), 1-10. doi:10.1016/S0168-1605(00)00377-9

3. Bruneel, F., Tubach, F., Corne, P., Megarbane, B., Mira, J. P., Peytel, E., Camus, C., Schortgen, F., Azoulay, E., Cohen, Y., Georges, H., Meybeck, A., Hyvernat, H., Trouillet, J.L., Frenoy, E., Nicolet, L., Roy, C., Durand, R., Le Bras., J., Wolff, M. \& Severe imported malaria in adults (SIMA) study group. (2010). Severe imported falciparum malaria: a cohort study in 400 critically ill adults. PLoS One, 5: e13236. doi: 10.1371/journal.pone.0013236.

4. Gravet, A., Couppie, P., Meunier, O., Clyti, E., Moreau, B., Pradinaud, R., Monteil, H. \& Prevost, G. (2001). Staphylococcus aureus isolated in cases of impetigo produces both epidermolysin A or B and LukE- LukD in $78 \%$ of 131 retrospective and prospective 
cases. Journal of Clinical Microbiology, 39 (12), 4349-4356. doi: 10.1128/JCM.39.12.4349-4356.2001.

5. Madhusoodanan, J., Seo, K. S., Park, J. Y., Gill, A. L., Waterhouse, J., Remortel, B., Bohach, G. \& Gill, S. R. (2007). Abstr. 107th Genetics meeting of American Society for Microbiology , Washington, DC, abstract, B-420.

6. McCormick, J. K., Yarwood, J. M., \& Schlievert, P. M., (2001). Toxic shock syndrome and bacterial superantigens: an update. Annual Review of Microbiology, 55, 77-104. DOI: 10.1146/annurev.micro.55.1.77.

7. Nanoukon, C., Argémi, X., Sogbo, F., Orekan, J, Keller, D., Affolabi, D., Schramm, F., Riegel, P., Baba-Moussa, L. \& Prévost, P. (2017). Pathogenic features of clinically significant coagulase-negative staphylococci in hospital and community infections in Benin. International Journal of Medical Microbiology, 307 (1), 75-82. doi: 10.1016/j.ijmm.2016.11.001.

8. Nyein, P. P., Aung, N. M., Kyi, T. T., Htet, Z. W, Anstey N. M., Kyi M.M. \& Hanson, J. (2016). High frequency of clinically significant bacteremia in adults hospitalized with falciparum malaria. Open Forum Infectious Diseases, 3 (1), ofw028. doi: 10.1093/ofid/ofw028.

9. Prévost G. (2004). Toxins in Staphylococcus aureus pathogenesis. In Proft M. (Ed.): Microbial toxins: molecular and cellular biology, Norfolk, England, Horizon Bioscience.

10. Riegel, P., Archambaud, M., Clavé, D., Vergnaud, M. (2006). Bactérie de culture et d'identification difficiles. Nancy l'Etoile, France, Biomérieux.

11. Salgado-Pabón, W., Breshears, L., Spaulding, A. R., Merriman, J. A., Stach, C. S., Horswill, A. R., Peterson, M. L. \& Schlievert, P. M. (2013). Superantigens are critical for Staphylococcus aureus infective endocarditis, sepsis, and acute kidney injury. Mbio, 4: e00494-13. doi 10.1128/mBio.00494-13

12. Sandlund, J., Naucler P, Dashti, S, Shokri, A., Eriksson, S., Hjertqvist, M., Karlsson, L., Capraru, T. \& Färnert, A. (2013). Bacterial co-infections in travelers with malaria: rationale for antibiotic therapy. Journal of Clinical Microbiology, 51 (1), 15-21. doi: 10.1128/JCM.02149-12

13. Scott, J. A., Berkley, J. A. Mwangi, I., Ochola, L., Uyoga, S., Macharia, A, Ndila, C, Lowe, B. S., Mwarumba, S, Bauni, E, Marsh, K. \& Williams, T. N. (2011). Relation between falciparum malaria and bacteremia in Kenyan children: a population-based, case-control study and a longitudinal study. Lancet, 378 (9799), 1316-1323. doi: 10.1016/S0140-6736(11)60888-X. 
14. Spaulding, A.; Salgado-Pabón, W.; Kohler, P.; Horswill, A.; Leung D. \& Schlievert, P. (2013). Staphylococcal and streptococcal superantigen exotoxins. Clinical Microbiology Reviews, 26 (3), 422447. doi: 10.1128/CMR.00104-12.

15. Were, T., Davenport, J. B., Hittner, C., Ouma, J. M., Vulule, J. M., Ong'echa, M. J., D. J. \& Perkins, D. J. (2011). Bacteremia in Kenyan children presenting with malaria. Journal of Clinical Microbiology, 49 (2), 671676. doi: $\underline{10.1128 / J C M .01864-10}$ 